\title{
Determination of Detectable Fatigue Crack Length by Improved AHP Method for Civil Aircraft Structures
}

\author{
Baohui Jia \\ Aeronautical Engineering Institute \\ Civil Aviation University of China \\ Tianjin, China \\ jiabaohui@sina.com
}

\author{
Shuai Tong \\ Aeronautical Engineering Institute \\ Civil Aviation University of China \\ Tianjin, China \\ sosots0421@gmail.com
}

\author{
Jun Xue \\ Aeronautical Engineering Institute \\ Civil Aviation University of China \\ Tianjin, China \\ 18665454306@163.com \\ Xiang Lu \\ Aeronautical Engineering Institute \\ Civil Aviation University of China \\ Tianjin, China \\ xlu5855@hotmail.com
}

\begin{abstract}
In order to ensure the safety and reliability of civil aircraft structures, the present paper focus on determination of detectable fatigue crack length by improved Analytic Hierarchy Process (AHP) method for civil aircraft structures. By rating the impact of various factors, fatigue damage such as detectable cracks are considered to establish a rating system, and apply improved AHP for overall ratings each index draw total level. On this basis, according to the regression equation of total level and basically detectable crack length derived the size of basically detectable crack. In the example of this paper, the basically detectable crack length derived by the improved method compared to the length derived by unimproved method shortened $2 \mathrm{~mm}$. The result indicated that the improved method can avoid overly conservative due to maintenance intervals developed and save maintenance costs. This research provides a theoretical basis for airlines to develop an economic and reasonable structural fatigue damage inspection interval according to the actual situation of the aircraft.
\end{abstract}

Keywords-Aircraft Structure; fatigue damage; basically detectable crack; analytic hierarchy process; inspection interval

\section{INTRODUCTION}

In order to ensure the safety and reliability of civil aircraft, the maintenance review board report must be formulated before new civil aircraft put into use $\mathrm{e}^{[1]}$. Maintenance review board report (MRBR) is also called maintenance requirements or maintenance technical regulations. It is the based primarily on maintaining the continuing airworthiness of aircraft and the basic documents for the development of maintenance programs and work cards by aircraft carriers ${ }^{[2]}$.

MSG-3 analysis method was widely used in the current to develop MRBR. The analysis portions of MSG-3 consists of four main parts, including (1)Aircraft Structures; (2) Systems/Powerplant; (3) Zonal Inspections; (4)Lightning/High Intensity Radiated Field. Aircraft structures need to be evaluated by accidental damage (AD), environmental deterioration (ED), fatigue damage (FD) and to develop corrosion prevention and control program(CPCP) when develop structural MRBR.

In order to determine the time to inspect the aircraft with what kind of level of inspection, and then to decide which type of maintenance should be performed. These are the core issues to develop $\mathrm{MRBR}^{[3]}$. For fatigue damage of metal structures, how to determine inspection intervals that are reasonable need to be considered when formulating the structural MRBR. When using the visual inspection method, first of all basic crack of structure must be determined, and then obtained the length of detectable crack by visual inspection. The time of crack growth is analysised according to the length of hidden crack and the growth curve of fatigue crack. The first inspection threshold with corresponding check type can be calculated. Finally, complete the evaluation of fatigue damage of initial MRBR.

At present, the evaluation of fatigue damage of initial MRBR for main aircraft models generally use the method "Determine the evaluation index - Index rating Comprehensive indexes - Determine the interval" ideas. The methods of rating each index and comprehensive indexes are the core and foundation of the whole evaluation process.

Engineering practice is commonly used to rating each index. The methods of Comprehensive indexes rating include: mean rating method, lowest rating method, matrix rating method and transitional rating method. The matrix rating method is most commonly used among them, but the influence and impact on fatigue damage inspection of each index is not considered in these methods ${ }^{[4]}$. 
Hierarchical analysis method can determine the weight effectively ${ }^{[5]}$, but it is has two obvious deficiencies in the algorithm: firstly, AHP only emphasizes the data itself, ignoring the correction data between each other, lost some potential correction information. Secondly, solving eigenvalues and eigenvectors of matrix are complex, and at present the approximate solution method are widely used but the accuracy of the result is seriously affected. In this paper, the AHP algorithm has been improved, and the calculation process is simplified, so that the total level is more in line with established engineering practice.

\section{SIZE RATING METHOD OF THE BASIC DETECTABLE CRACK}

\section{A. Classification analysis of various indexes}

Civil aircraft structural fatigue damage is caused by the cyclic loading and continuously superimposed which include crack initiation and crack propagation ${ }^{[6]}$. This damage is a cumulative process and related to the use of the aircraft (flight hour or flight cycle) ${ }^{[7]}$. For metal structural fatigue damage, the analysis of basic detectable crack length needs to evaluate the detectability of each fatigue damage crack of structure before reaching the critical value $a_{c}$. The factors that affect the detectability of fatigue damage of structure include: visibility, crack density, size of structure, lighting conditions, surface conditions etc ${ }^{[8]}$. The model shown in Fig .1.

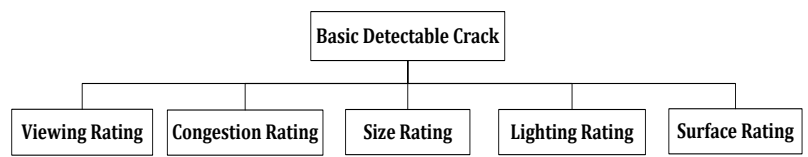

Figure 1. Factors affect visual inspection of basic detectable cracks identified.

The level of structure size is determined by the size of inspection zone and the size of structural significant item. First of all, divide the size of zone and structural significant item (SSI) as shown in TABLE I:

TABLE I. CLASSIFICATION OF RATING SIZE OF ZONE AND STRUCTURAL SIGNIFICANT ITEM

\begin{tabular}{ccc}
\hline Level & Dimensions of Zone & Dimensions of SSI \\
\hline Small & Dimensions of zone is & $\begin{array}{c}\text { Small-sized parts, not } \\
\text { more than } 10 \mathrm{~cm}^{2}\end{array}$ \\
Medium & approximately $1 \mathrm{~m}^{2}$ or even & Medium-sized parts \\
& smaller & \\
Large & ie: the wings and intact skin & $\begin{array}{c}\text { Large-sized parts, for } \\
\text { example, bulkheads, } \\
\text { spars, etc }\end{array}$ \\
\hline
\end{tabular}

Size classification is shown in TABLE II, and generally divided into four ratings.
TABLE II.

ZONE SIZE RATING

\begin{tabular}{cc}
\hline Rating & Configuration Item /Rating of Zonal Dimension \\
\hline 1 & Zones with large level \\
2 & Zones with medium level or configuration items \\
3 & with lager level \\
4 & Configuration items with medium level \\
& small level \\
\hline
\end{tabular}

The rating of viewing depends on the distance from structural inspection items to eyes of inspector; the rating of congestion depends on the number of equipment components and complexity within the inspection zone; the rating of lighting depends on the light source and light quality; the rating of surface depends on coating properties and the use of sealants and cleaners. All of the factors are classified in TABLE III, and usually divided into four ratings from $0-3$.

TABLE III. THE CLASSIFICATION OF VISIBILITY, DENSITY, LIGHTING AND SURFACE

\begin{tabular}{|c|c|c|c|c|}
\hline & Level 0 & Level 1 & Level 2 & Level 3 \\
\hline Viewing & $\begin{array}{l}\text { "Inaccessible } \\
\text { concealed } \\
\text { items or the } \\
\text { distance from } \\
\text { structure to } \\
\text { inspector's } \\
\text { eyes more } \\
\text { than } 300 \mathrm{~cm}\end{array}$ & $\begin{array}{l}\text { "Bad"- } \\
\text { the distance } \\
\text { from } \\
\text { structure to } \\
\text { inspector's } \\
\text { eyes between } \\
150 \mathrm{~cm}- \\
300 \mathrm{~cm}\end{array}$ & $\begin{array}{l}\text { "medium"- } \\
\text { - the } \\
\text { distance from } \\
\text { structure to } \\
\text { inspector's } \\
\text { eyes between } \\
50 \mathrm{~cm}-150 \mathrm{~cm}\end{array}$ & $\begin{array}{l}\text { "Good"- } \\
\text { No limit or } \\
\text { the distance } \\
\text { from } \\
\text { structure to } \\
\text { inspector's } \\
\text { eyes is close } \\
\text { enough }\end{array}$ \\
\hline Congestion & - & Dense & Medium & $\begin{array}{r}\text { Not } \\
\text { dense }\end{array}$ \\
\hline Lighting & & $\begin{array}{l}\text { Structure or } \\
\text { zone to be } \\
\text { inspected in } \\
\text { the shadow } \\
\text { area } \\
\text {, for } \\
\text { example, } \\
\text { landing gear } \\
\text { pods without } \\
\text { direct light } \\
\text { source }\end{array}$ & $\begin{array}{l}\text { The outer } \\
\text { surface with } \\
\text { adequate } \\
\text { lighting and } \\
\text { the internal } \\
\text { structure of } \\
\text { the aircraft } \\
\text { with artificial } \\
\text { lighting }\end{array}$ & $\begin{array}{c}\text { There is } \\
\text { centralized } \\
\text { lighting when } \\
\text { inspect }\end{array}$ \\
\hline Surface & & $\begin{array}{l}\text { The zones or } \\
\text { items easily } \\
\text { to be covered } \\
\text { by sealant or } \\
\text { suffer too } \\
\text { much fat, } \\
\text { fuel or dust } \\
\text { pollution }\end{array}$ & $\begin{array}{c}\text { Clean zones } \\
\text { or items }\end{array}$ & - \\
\hline
\end{tabular}


According to the classification standards above and combine with the utilization of aircraft and work experience, engineers can evaluate the specific level of each factor.

\section{B. Using the improved AHP method to determine the weight of each factor}

After obtain the level of each factor, a calculation method was adopted to synthesize various indicators to form a total level. This paper uses the improved AHP to synthesize each index. The improved analysis procedure is as follows:

1) Determine the evaluation index and establish hierarchy. Hierarchical model of this paper was shown in Fig.1.

\section{2) Establishing Judgment matrix.}

Assuming the problem $B$ is determined by $n$ elements, $b_{1}, b_{2}, b_{3}, \ldots, b_{n}$, mark $B=\left\{b_{1}, b_{2}, b_{3}, \ldots, b_{n}\right\}$. The weight of each element is set as $q_{1}, q_{2}, \ldots, q_{n}$, $Q=\left\{q_{1}, q_{2}, \ldots, q_{n}\right\}$. Mark matrix $A=\left\{a_{i j}\right\}=\left\{q_{i} / q_{j}\right\}$. Matrix $A$ meets the condition of $a_{i i}=1, a_{i j}=1 / a_{j i}$ and $a_{i j}=a_{i k} / a_{j k}=a_{i k} \cdot a_{k j}(i, j, k=1,2, \ldots, n)$, so it meets the condition of complete consistency ${ }^{[9]}$.

Assuming $t_{i j}$ is the priority that $b_{i}$ compare to $b_{j}$, and mark matrix as $T=\left\{t_{i j}\right\}$ formed by the $t_{i j}$. There are two ways to establish judgment matrix: one is the 1-9 scaling method that scale by expert scoring or statistics. Another way is apply rough set theory to determine the importance of each factor, then establish judgment matrix by the importance of each factor. The element $t_{\mathrm{ij}}$ of judgment matrix $T$ that established by two ways above is not the value of $q_{i} / q_{j}$, because $q_{i}$ is the exact solution of importance of each factor in the hypothesis. The above two ways just combining expert scoring and engineering experience to get the estimated value of $q_{\mathrm{i}} / q_{\mathrm{j}}$. Then use the estimated value to establish judgment matrix. But the judgment matrix obtained by this method is not precise enough, hence the judgment matrix need for further modification.

\section{3) The modification of judgment matrix $T$}

The previous judgment matrix $T$ does not meet the condition for complete consistency, only meets $t_{i i}=1$, $t_{i j}=1 / t_{j i}$. So judgment matrix $T$ need to be modified. $t_{i j}$ is an approximate estimated value of $q_{i} / q_{j}$. Thus, for each $k$ $(k=1,2, \ldots, \mathrm{n})$, approximate estimated value of $q_{i} / q_{j}$ is also $t_{i k} / t_{j k}=t_{i k} \cdot t_{k j}$. .Then take the geometric mean value of

them and mark as $c_{i j}^{(1)}, c_{i j}^{(1)}=\sqrt[n]{\prod_{I=1}^{n} t_{i I} t_{I j}} c_{i j}^{(1)}$ is the new approximate estimated value of $q_{i} / q_{j}$. According to this method calculate $N$ times, the limit valve is the best estimated value of $q_{\mathrm{i}} / q_{\mathrm{j}}$ when limit valve convergence. The proof of the limit is convergence, and only once iterations by geometric average reached the limit and the limit value is $c_{i j}^{(1)}$. Mark the modified matrix as $C=\left\{c_{i j}\right\}$, in this case the corresponding eigenvalue of the matrix $C$ is $n$ and meets the consistency condition.

\section{4) Determine the weight of each factor}

The eigenvector corresponding to the largest eigenvalue is $\omega=\left\{\omega_{i}\right\}$ by the modified matrix $C$ and $\omega_{i}=\sqrt[n]{\prod_{j=1}^{n} c_{i j}}$

$(i=1,2, \ldots, n)$. The weights of each factor can be obtained after $\omega=\left(\omega_{i}\right)^{\mathrm{T}}$ be normalized.

The modified matrix generally meets the consistency condition. In order to verify the correctness of the improved algorithm, need to verify its consistency, the verification method is the same as the method of not improved analytic hierarchy process, use $C R=C I / R I$ to express good or bad of quality of consistency. When $C R<0.1$, shows good consistency with the matrix after modification.

\section{Determine the total level and the size of basic detectable crack}

When use analytic hierarchy process to determine the total level of damage, the size of basic detectable crack of fatigue damage can be considered as target layer. Viewing rating, congestion rating, size rating, lighting rating, and surface rating can be considered as the index of index layer, then calculate the weight of each index. After obtaining the weights of each factor, the total level of fatigue damage can be obtained by weighted sum.

Set $W$ as total level of fatigue damage, $W_{1}$ as rating of viewing, $W_{2}$ as rating of congestion, $W_{3}$ as rating of size, $W_{4}$ as rating of lighting, $W_{5}$ as rating of surface, and set the weight $q_{1}, q_{2}, q_{3}, q_{4}, q_{5}$ as each index corresponding. The total level $W$ is calculated as shown in (1):

$W=\left(W_{1}, W_{2}, W_{3}, W_{4}, W_{5}\right) \cdot\left(q_{1}, q_{2}, q_{3}, q_{4}, q_{5}\right)^{\mathrm{T}}$

The current total level and the chart of basic crack size did not consider the impact on the total level of each index. The size of detectable crack can be calculated by the regression equation corresponding to the size of basic detectable crack of fatigue damage $L_{\mathrm{BAS}}$ and total level $W$ established by the literature [10]. The regression equation as shown in (2):

$$
\begin{aligned}
L_{\mathrm{BAS}}= & -28.32 W^{5}+294 W^{4}-1184.9 W^{3}+2387.4 W^{2}- \\
& 2622.9 W+1450.3
\end{aligned}
$$

That $W$ is the total level and $L_{\mathrm{BAS}}$ is the size of basic detectable crack. (Unit: $\mathrm{mm}$ )

The size of visual detectable crack can be calculated by the size of basic detectable crack, and combined with the size of covert crack and the fatigue crack growth curve, the initial inspection threshold of fatigue damage can be calculated.

\section{RATING THE INSPECTION INDEX OF CRACK OF OUTER WING LEADING EDGE}

Select the outer wing leading edge rear surface of a certain type of aircraft as an example to analysis by improved AHP in details. The outer wing leading edge rear surface is an entire wing skin. There are certain amount and complex components in this region without direct light source. The distance between inspectors to this region is from $150 \mathrm{~cm}$ to $300 \mathrm{~cm}$, and easier to be covered by the sealant, oil, fuel or dust pollution to effect the check of this region. The check indexes of basic detectable crack of fatigue damage for the outer wing leading edge rear 
surface are rated by the utilization of aircraft and engineering experience. The rating results as shown in TABLE IV:

TABLE IV. EVALUATION RATING INDEXES

\begin{tabular}{c|ccccc}
\hline & $\begin{array}{c}\text { Viewing } \\
\text { Rating } W_{1}\end{array}$ & $\begin{array}{c}\text { Congestion } \\
\text { Rating } W_{2}\end{array}$ & $\begin{array}{c}\text { Size Rating } \\
W_{3}\end{array}$ & $\begin{array}{r}\text { Lighting } \\
\text { Rating } W_{4}\end{array}$ & $\begin{array}{c}\text { Surface } \\
\text { Rating } W_{5}\end{array}$ \\
\hline Rating & 1 & 2 & 1 & 1 & 1 \\
\hline
\end{tabular}

The judgment matrix $T$ is constructed in literature [10]:

$$
T=\left[\begin{array}{ccccc}
1 & 1.1588 & 1.3831 & 1.2597 & 0.8052 \\
0.8629 & 1 & 1.1966 & 1.0899 & 0.6966 \\
0.7230 & 0.8357 & 1 & 0.9108 & 0.5822 \\
0.7938 & 0.9175 & 1.0979 & 1 & 0.6392 \\
1.2419 & 1.4355 & 1.7176 & 1.5645 & 1
\end{array}\right]
$$

Let the matrix $T$ self-coordination by the improved algorithm to get the completely consistent matrix $C=\left(c_{i j}\right)_{5 \times 5}$ $c_{i j}=\sqrt[5]{\prod_{I=1}^{5} t_{i I} t_{I j}}$.

$$
C=\left[\begin{array}{ccccc}
1 & 1.1570 & 1.3838 & 1.2604 & 0.8056 \\
0.8643 & 1 & 1.1960 & 1.0893 & 0.6963 \\
0.7226 & 0.8361 & 1 & 0.9108 & 0.5822 \\
0.7934 & 0.9180 & 1.0979 & 1 & 0.6392 \\
1.2413 & 1.4362 & 1.7176 & 1.5645 & 1
\end{array}\right]
$$

Verify the consistency of matrix $C$. Characteristic value $\lambda_{\max }=5.0001, C I=\left(\lambda_{\max }-n\right) /(n-1)$ ( $n$ is the rank of matrix $C$ ). Since $C I \approx 0$ of matrix $C$, then it can be considered to be completely consistent. Thus, the characteristic vector as follows:

$$
\begin{aligned}
& \omega_{1}=\sqrt[5]{\prod_{I=1}^{5} c_{1 I}}=\sqrt[5]{1 \times 1.1570 \times 1.3838 \times 1.2604 \times 0.8056}=1.1021 \\
& \omega_{2}=\sqrt[5]{\prod_{I=1}^{5} c_{2 I}}=\sqrt[5]{0.8643 \times 1 \times 1.1960 \times 1.0893 \times 0.6963}=0.9525 \\
& \omega_{3}=\sqrt[5]{\prod_{I=1}^{5} c_{3 I}}=\sqrt[5]{0.7226 \times 0.8361 \times 1 \times 0.9108 \times 0.5822}=0.7964 \\
& \omega_{4}=\sqrt[5]{\prod_{I=1}^{5} c_{4 I}}=\sqrt[5]{0.7934 \times 0.9180 \times 1.0979 \times 1 \times 0.6392}=0.8744 \\
& \omega_{5}=\sqrt[5]{\prod_{I=1}^{5} c_{5 I}}=\sqrt[5]{1.2413 \times 1.4362 \times 1.7176 \times 1.5645 \times 1}=1.3680
\end{aligned}
$$

After normalization to get the weight of each index, $q_{1}=0.2164, q_{2}=0.1870, q_{3}=0.1564, q_{4}=0.1717, q_{5}=0.2686$.

After obtaining weight of each index and level of basic detectable crack of fatigue damage, put them into (1), then obtain the total level of basic crack length $W=1.1871$.Put $W$ into (2) to obtain the size of basic detectable crack $L_{\mathrm{BAS}}=234.32(\mathrm{~mm})$.

The size of basic detectable crack comparison table from reference [8] is shown in TABLE V. When viewing rating is 1 , congestion rating is 2 , size rating is 1 , lighting rating is 1, surface rating is 1, the corresponding practicality rating is 1 and corresponding condition rating is 1 . In this case the detectable crack size is $295 \mathrm{~mm}$. The

\begin{tabular}{|c|c|c|c|c|c|}
\hline & \multicolumn{4}{|c|}{ Condition Rating } \\
\hline & & 1 & 2 & 3 & 4 \\
\hline \multirow{5}{*}{ 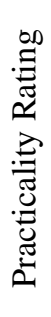 } & 1 & 295 & 205 & 145 & 100 \\
\hline & 2 & 205 & 100 & 70 & 50 \\
\hline & 3 & 145 & 70 & 35 & 22 \\
\hline & 4 & 100 & 50 & 15 & 10 \\
\hline & 5 & 70 & 22 & 10 & 8 \\
\hline
\end{tabular}
detectable crack size calculated by improved algorithm is between $205 \mathrm{~mm}$ and $295 \mathrm{~mm}$. Thus, the results that calculated by improved algorithm in line with the actual situation.

TABLE V. THE SIZE OF BASIC DETECTABLE CRACK CONTRASTIVE TABLE (UNIT: MM)

In order to compare the improved algorithm and the algorithm without improved, determine the index rating and basic detectable crack size for the above structure with unimproved analytic hierarchy process. Results are shown in TABLE VI:

TABLE VI. THE RESULTS CONTRASTIVE BETWEEN IMPROVED AND UNIMPROVED ALGORITHM

\begin{tabular}{ccc}
\hline & Rating & $\begin{array}{c}\text { Size of Basic Detectable } \\
\text { Crack }(\mathrm{mm})\end{array}$ \\
\hline Improved AHP & 1.1847 & 236.6 \\
Unimproved AHP & 1.1871 & 234.3 \\
\hline
\end{tabular}

According to the calculation results from TABLE VI, the difference between improved AHP and unimproved AHP is about $2 \mathrm{~mm}$. To determine the maintenance interval in late stage, $2 \mathrm{~mm}$ differences may lead to big difference of maintenance interval. By improving the maintenance interval to avoid making maintenance interval conservative and increasing repair costs. This is just determine the detectable crack size which corresponding to the general visual inspection, did not study the detectable crack size which corresponding to higher level visual inspection. In the above example, the crack size is not the crack size of actual structure, but the visual detectable crack size corresponding to general visual inspection. Obviously when the crack is extended to $234.3 \mathrm{~mm}$ the structure has already exceeded the fatigue critical crack length. This shows that the use of general visual inspection is not appropriate, the detectable crack size corresponding to higher-level way of checking need further analysis.

\section{CONCLUSIONS}

(1) The weights determine method by improved AHP consider the impact of each factor on the comprehensive rating of fatigue damage assessment. By this method, the determination of basic detectable crack size is more reasonable, and it also can provide a theoretical basis for later develop inspection intervals. Airlines can arrange inspection and repair according to the actual use of the 
aircraft, to determine a reasonable and efficient repair work card.

(2) The improved AHP modified the judgment matrix, considering the potential correction information between each influence factor; make the judgment matrix as consistency matrix. At the same time, the improved algorithm simplifies the process of calculation of fatigue damage assessment, improve efficiency and make the evaluation results more consistent with practical. It can provide a scientific and reasonable basis for later develop maintenance interval, avoid the maintenance interval not too conservative and save repair cost, which is important to airlines to reduce the operation cost.

\section{ACKNOWLEDGMENT}

The authors would like to acknowledge the financial support received from the National Natural Science Foundation of China, project "The Research of Civil Aircraft Maintenance Management Scheduling Optimization Method Based on Intelligent Algorithm" (U1233107), and the Central Universities Foundation of China, pre-research of major project "The Research of Key Technology for Maintenance Engineering Analysis"(3122014P002)

\section{REFERENCES}

[1] Shiji Chang. Modern Civil Aviation Maintenance Engineering Management[M]. Taiyuan: Shanxi Science and Technology Publishing House, 2002.

[2] Hongfu Zuo, Jing Cai, Hao Wu, etc. Aviation Maintenance Engineering[M]. Beijing: Science Press, 2011.

[3] ATA MSG-3 Operator/Manufacturer Scheduled Maintenance Developments. Air transport association of America, 2009.

[4] Hao Wu, Hongfu Zuo, Wei Sun. A Study on the Accidental Damage Inspection Intervals of Aircraft Structure Based on the Improved AHP[J]. Aircraft Design, 2008, 28(3): 58-59.

[5] Li Niu, Ke Chen, Yuan Cheng. Application of Improved AHP in Employment Comprehensive Evaluation[J]. Computer Simulation, 2011, 28(5):376-379.

[6] Xiaoling Zheng. Durability and Damage Tolerance Design for Structures of the Civil Aircraft (Volume 1): Fatigue Design and Analysis[M]. Beijing: Aviation Industry Press, 2003

[7] He Lin. The Research of Structure Damage Assessment Method for Civil Aircraft Maintenance Program[D]. Nanjing: Nanjing University of Aeronautics and Astronautics, 2006.

[8] A318/319/320/321 maintenance program development policy and procedures handbook: Airbus Company, 2001

[9] Yanping Jiang, Zhiping Fan. The decision theory and methods based on judgment matrix[M]. Beijing: Science Press, 2008.

[10] Haibin Yang. The Research of Maintenance Decision and Assessment Method for Aircraft Structural Fatigue Damage[D] Nanjing: Nanjing University of Aeronautics and Astronautics, 2011. 